\title{
Study of Generating Sub-Diffraction-Limit Patterns with the Phase Modulation of Polarized Beams
}

\author{
Chen Zhang ${ }^{1}$, Hongfu $\mathrm{Li}^{2}$, Shuang Wang ${ }^{1}$, Wei Zhao ${ }^{1}$, Xiaoqiang Feng ${ }^{1}$, Kaige Wang ${ }^{1}$, Guiren Wang ${ }^{3}$ and Jintao Bai ${ }^{1}$ \\ ${ }^{1}$ Institute of Photonics \& Photon-Technology, Northwest University, Xi'an, Shaanxi 710069, China \\ E-mail: wangkg@nwu.edu.cn \\ ${ }^{2}$ Kunming Institute of Physics, Kunming, Yunnan 650223, China \\ ${ }^{3}$ Mechanical Engineering Department \& Biomedical Engineering Program, University of South Carolina, Colum- \\ bia SC 29208, USA \\ In this work, laser spatial patterns with sub-diffraction-limit structures are acquired systematically by wave- \\ front modulation of different polarized Gaussian beams based on the vectorial diffraction theory. The \\ Gaussian beams, which are in circular, radial and azimuthal polarization, are applied with special designed \\ phase plates. With the modification of the phase factor in diffraction integral formula, new kinds of phase \\ plates are carried out and employed with the polarized beams. The mechanism of forming sub-diffraction- \\ limit patterns has been illustrated detailedly, as well as the topography of the vast novel beam patterns with \\ special dimensional distribution near the focal plane. The special wavefront designing of Guassian light \\ beam provides many useful beam patterns which have great potential in the areas of far-field nanoscope, \\ optical tweezer and beam fabrication.
}

DOI: 10.2961/jlmn.2016.03.0002

Keywords: sub-diffraction-limit, beam patterns, diffraction theory, polarization, phase modulation

\section{Introduction}

Recently, sub-diffraction-limit laser patters, which are generally acquired by the temporal and spatial modulating of Gaussian beam wavefront, have attracted great interest for their outstanding performance in front-line science researches [1-4]. For instance, the doughnut-shaped beam pattern, which contains a null intensity center, is playing a critical role in the Stimulated Emission Depletion (STED) nanoscope[5,6]. The employment of this special shaped laser beam makes the far-field microscope reaches a lateral resolution of $5.8 \mathrm{~nm}$ [7-10]. This kind of sub-diffractionlimit beam pattern is also used as the core technique of optical tweezers to stably trap the low-index or nontransparent particles in a two-dimension structure [11]. While, it also contributed to the nano pillars array fabrication with pillars' diameter less than 50nm [12].

Up to now, the special sub-diffraction-limit beam patterns can be obtained by utilizing phase-plate [13,14], Spatial Light Modulator (SLM) $[15,16]$, and Vector Superposition [17-20]. Among all these methods, the modulation employing phase plate is the most cost-saving, convenient and effective for creating sub diffraction limitation beam pattern. Dr. Singh's group has analyzed the topography of Laguerre-Gaussian beam with different topological charge $[21,22]$. Dr. Miyamoto have studied the phase distortion characterizing and compensating in holograms to create a donut-shaped pattern [23]. Our group has also revealed the influence of phase distortion on the sub-diffraction-limit beam patterns [24]. However, there still lays plenty space for exploring more sub-diffraction-limit patterns which possess useful spatial structures and is capable to improve the performance of the laser $[14,25]$. The polarization of incident beam have a direct influence on focusing beam pattern in a high NA optical system. It would be a promising technology to generate beam patterns by combining the polarized Gaussian beams with designed phase plates, which might create diverse of useful beam patterns.
In this work, based on the vectorial diffraction theory, a systematical numerical simulation on exploring subdiffraction-limit spatial laser focal patterns will be carried out. The Gaussian beams with circular, radial and azimuthal polarization are modulated by special designed phase plates. The formation of the sub-diffraction-limit patterns has been analyzed and revealed in detail. The spatial distribution of the sub-diffraction-limit patterns are presented in focal space. They are capable to improve the performance of laser beam of nanoscope, optical tweezer, laser writing and other fields.

\section{Theory}

The electronic field distribution can be mathematically described by vector integral. Fig.1(a) is the schematic diagram of generating spatial beam patterns with the employment of different polarized beams and phase plates. The polarizations are circular, radial and azimuthal. Four kinds of phase plates, which are in shaped of semi-circle, quartered circle, circular and vortex, are chosen. The NA of objective lens is assumed to 1.4. Fig.1(b) reveals the geometric diagram of focusing the modulated beam with objective.

According to the Vectorial Debye theory, in the case of focusing Gaussian beam with a high NA objective lens, the diffracted field of the point P (Fig.1(b)) near focus can be expressed in cylindrical coordinates as the following equation1 $[25,26]$ :

$$
\begin{array}{r}
E\left(r_{p}, \varphi_{p}, z_{p}\right)=\left[\begin{array}{c}
E_{X} \\
E_{y} \\
E_{z}
\end{array}\right]=\iint_{\Omega} \sqrt{\cos \theta} \cdot \sin \theta \cdot E(\theta, \varphi) \cdot V(\theta, \varphi) \cdot\left[\begin{array}{c}
P_{X} \\
P_{y} \\
P_{z}
\end{array}\right] \\
\cdot \varphi_{s}(\theta, \varphi) \cdot e^{i k n\left(z \cdot \cos \theta+r_{p} \cdot \sin \theta \cdot \cos \left(\varphi-\varphi_{p}\right)\right)} d \varphi d \theta
\end{array}
$$

Where, $\mathbf{E}\left(r_{p}, \varphi_{p}, Z_{p}\right)$ represents the electric field vector at the point $\mathrm{P}\left(r_{p}, \varphi_{p}, Z_{p}\right)$. 

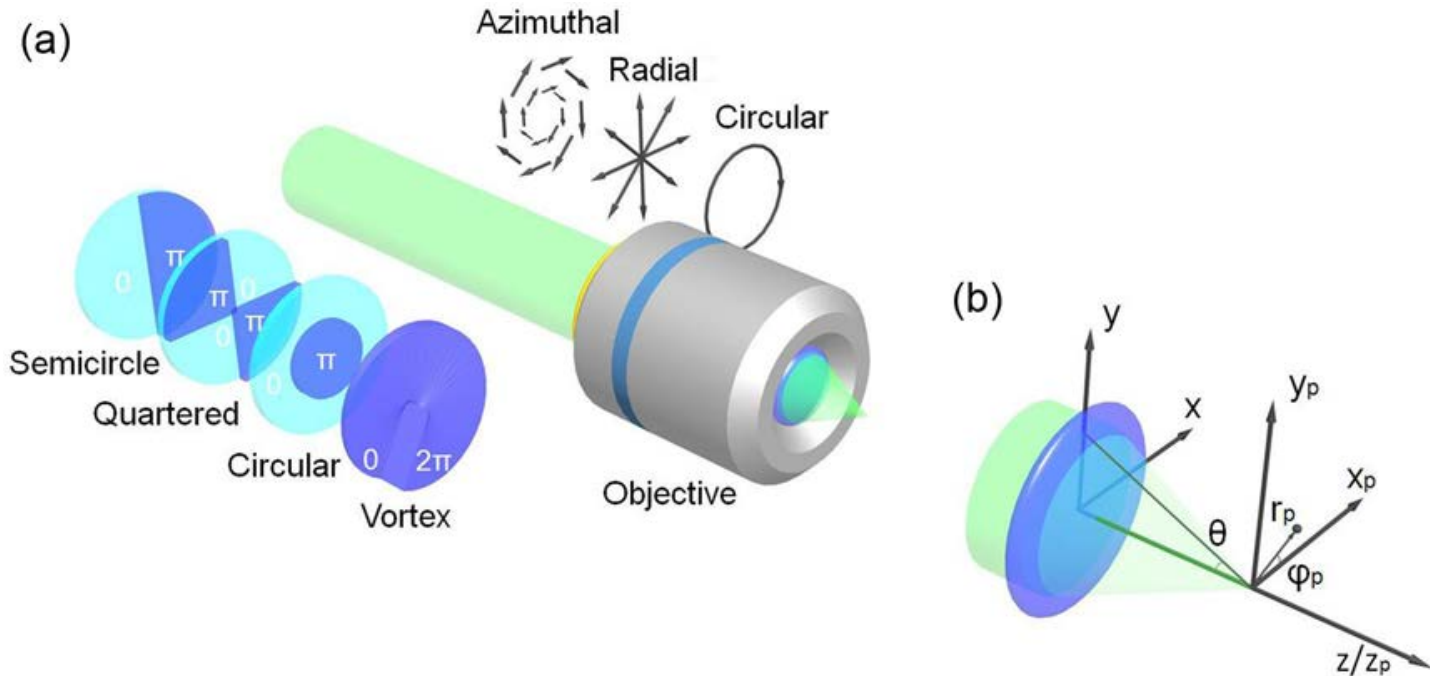

Fig.1 (a) Schematic of the optical system and (b) geometric diagram of light beam focusing.

$\theta$ is the angle between the laser direction and the optical axis, and $\varphi$ is the azimuthal angle; $E(\theta, \varphi)$ stands for the amplitude function of the input optical field, and $E(\theta$, $\varphi)=I_{0} \exp \left(-\gamma^{2} \sin ^{2} \theta / \sin ^{2} \theta_{\max }\right)$, where $I_{0}, \gamma$ and $\theta_{\max }$ are respectively the light intensity, truncation parameter and the maximal semi-aperture angle of objective lens; $\varphi_{\mathrm{s}}(\theta, \varphi)$ is the phase delay of phase plate; $n$ is the refractive index, $k=2 \pi / \lambda$ is the wave number; $\left[\mathrm{p}_{\mathrm{x}}, \mathrm{p}_{\mathrm{y}}, \mathrm{p}_{\mathrm{z}}\right]$ represents the matrix vector of the polarization of input laser, see Table 1 . The conversion matrix of the polarization from the object field to image field $\mathbf{V}(\theta, \varphi)$ is described as the equation 2 .

$$
V(\theta, \varphi)=\left[\begin{array}{ccc}
1+(\cos \theta-1) \cos ^{2} \varphi & (\cos \theta-1) \cos \varphi \sin \varphi & -\sin \theta \cos \varphi \\
(\cos \theta-1) \cos \varphi \sin \varphi & 1+(\cos \theta-1) \sin ^{2} \varphi & -\sin \theta \sin \varphi \\
\sin \theta \cos \varphi & \sin \theta \sin \varphi & \cos \theta
\end{array}\right]
$$

Table 1. Polarization vector matrix

\begin{tabular}{cccc} 
Polarization & Right circular & Radial & Azimuthal \\
\hline$\left[\begin{array}{c}P x \\
P y \\
P z\end{array}\right]$ & $\frac{1}{\sqrt{2}}\left[\begin{array}{l}1 \\
i \\
0\end{array}\right]$ & {$\left[\begin{array}{c}\cos \theta \\
\sin \theta \\
0\end{array}\right]$} & {$\left[\begin{array}{c}-\sin \theta \\
\cos \theta \\
0\end{array}\right]$} \\
\hline
\end{tabular}

\section{Numerical simulation}

The design of new phase plates is based on the combination of a circular phase plate with one of the other phase plates mentioned in Fig.1.

Fig.2 shows the phase plates used for wavefront designing, as well as the effect of the new phase plate on the wavefront of a flat Gaussian wave. As is illustrated in Fig.2, the phase of the incident wavefront is changed in the particular regions. The diameter of the Pi area in the Circular Pi phase plate is 0.707 times of the beam's diameter. The even change of phase from 0 to $2 \pi$ in clock-wise occurs in the Vortex 2Pi phase plate. It is shown obviously that, three types of new phase plates 1,2 and 3 are created by combining the circular Pi phase plate with the semi-circle $\mathrm{Pi}$, Quartered Pi and vortex 2Pi phase plates respectively.

Fig.3 reveals the mechanism of creating subdiffraction-limit patterns with the combination of polarized Gaussian beams and the designed phase plates. Right- handed, radial and azimuthal polarized Gaussian beams are employed with the type 1 to type 4 phase plates. As is illustrated in Fig.3, the arrows represents the temporal vibration direction of incident beam. By passing through the designed phase plates, the polarization are engineered to be changed properly to interfere with other rays in the focal space. The rays in opposite direction contribute to destructive interference in the beam patterns.

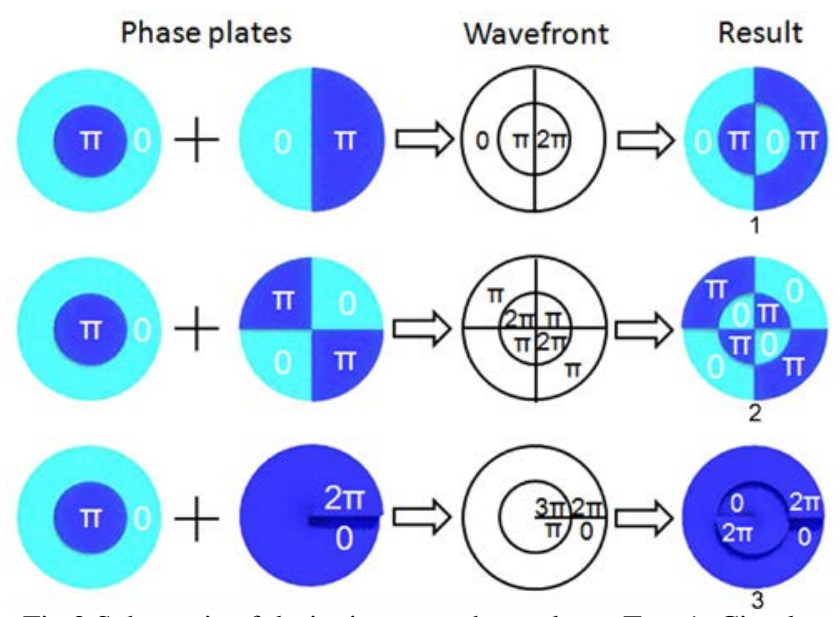

Fig.2 Schematic of designing new phase plates. Type1: Circular Pi\& Semi-circular Pi. Type 2: Circular Pi \& Quartered Pi. Type 3: Circular Pi \& Vortex 2Pi
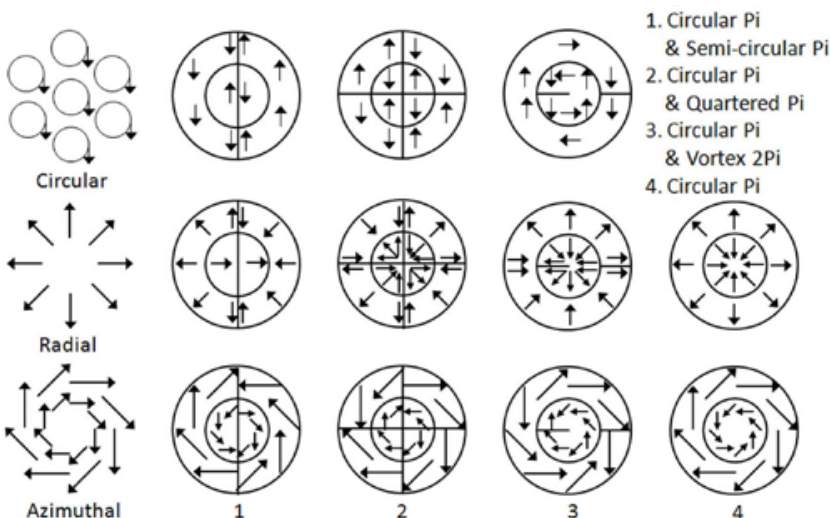

4. Circular Pi
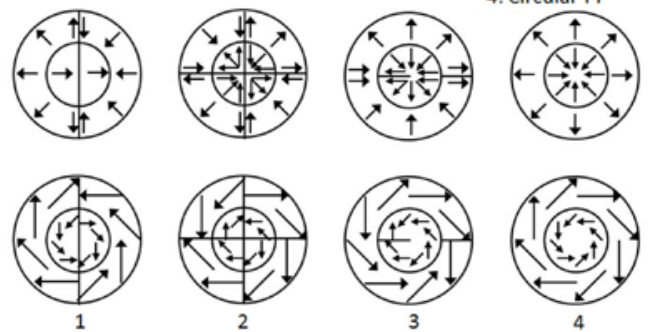

Fig.3 Mechanism of generating sub-diffraction-limit patterns with polarized Gaussian beam and designed phase plates. Type 4 : 
Circular Pi (Type 1 to 3 refer to the cases in Fig.2).

The diffraction integral formula is programmed by MATLAB to carry out the topography of sub-diffractionlimit patterns. In the calculation, the total amount of the
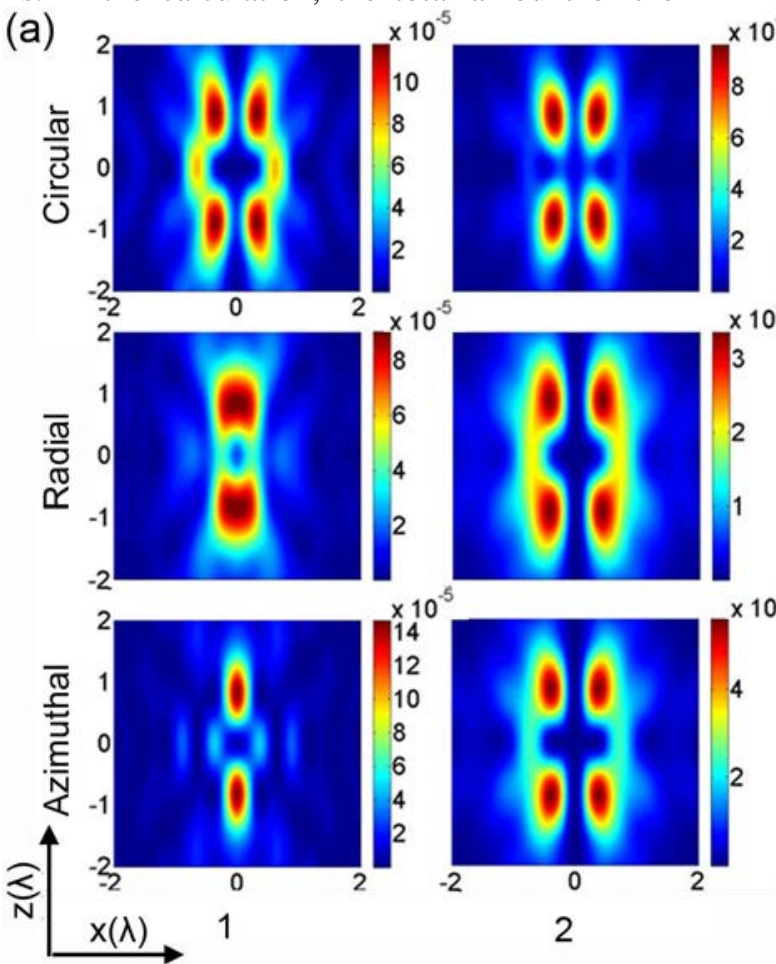

light intensity on incident plane is constant. All the simulation results are normalized by multiply the reciprocal of the whole intensity of the incident pupil.

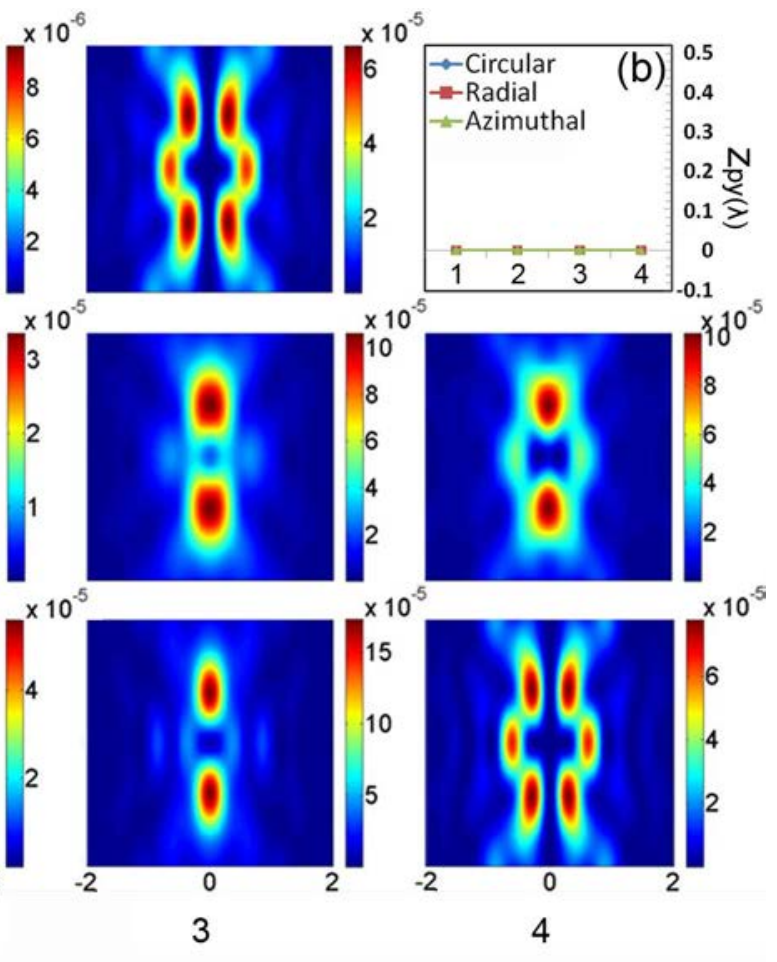

Fig.4 Intensity distributions of polarized Gaussian beam modulated with phase plates. (a) topography of sub-diffraction-limit patterns on axial direction; (b)position of axial calculation plane, $\mathrm{z}_{\mathrm{py}}=0$

(a)

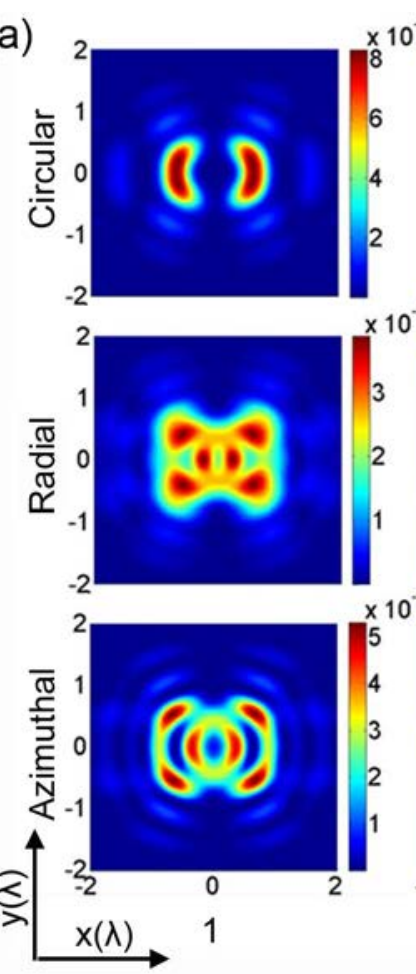

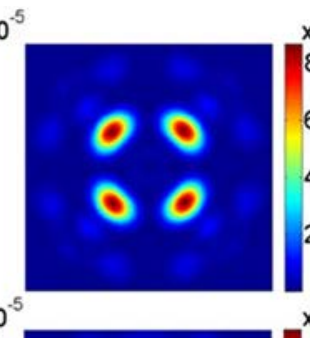
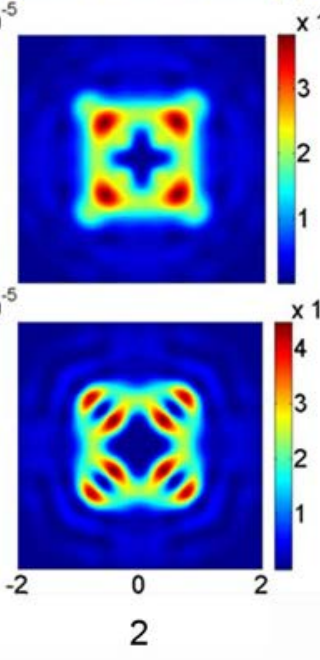
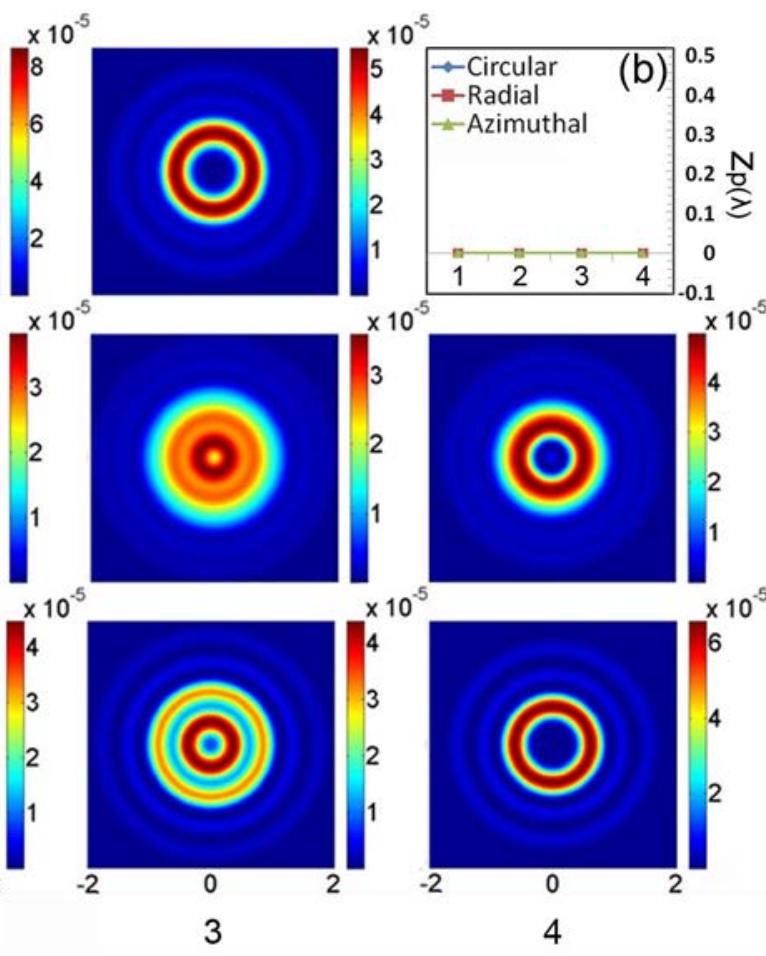

Fig.5 Intensity distributions of modulation carried out by polarized Gaussian beam with phase plates. (a) topography of sub-diffractionlimit patterns on lateral direction; (b)position of calculation plane, $\mathrm{z}_{\mathrm{py}}=0$

Fig.4 presents the axial intensity distributions of the different polarized Gaussian laser beams modulated with designed phase plates. In Fig.4(a), the polarizations from first row to third row are circular, radial and azimuthal re- spectively. The digit 1 to 4 refer to the phase plates mentioned in Fig.3. Fig.4(b) indicates that the calculation of all these modulation cases are carried out on xz plane. From the axial distribution of intensity, it can be clearly saw that 
plenty of the modulated Gaussian beams are created with particular spatial structures in axial dimension. It is worth to mention that, in the cases of radial polarized beam modulated with phase plate 1,3 and 4, as well as, azimuthal polarized beam applied with phase plate 1 and 3 , a subdiffraction-limit dark spot is created in the laser spot center. These structures seems capable for the usage of compressing the fluorescence PSF both in lateral and axial direction in the STED nanoscope; the hollow structures might helpful for atom capture as well.
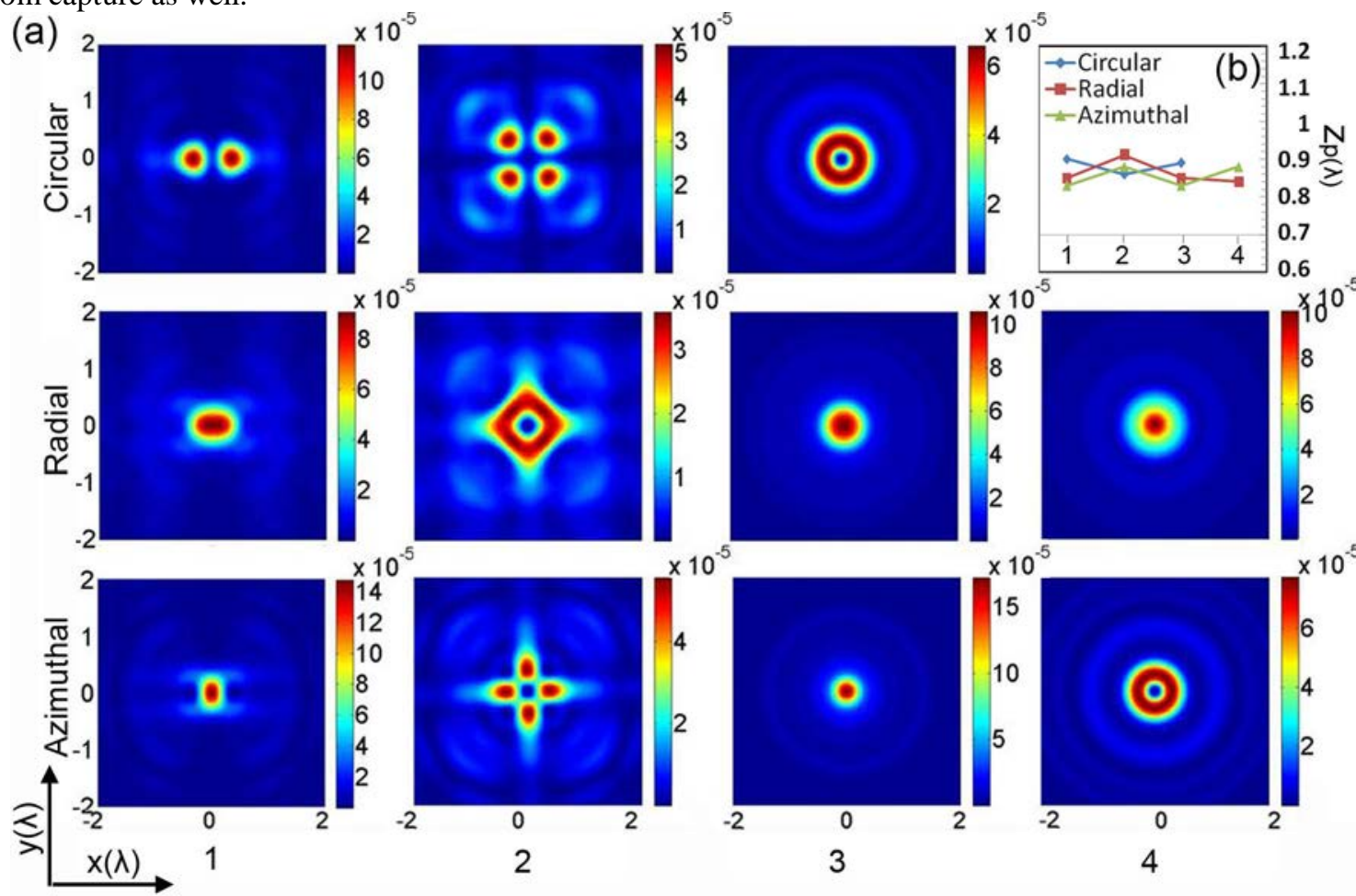

the plane of $\mathrm{z}_{\mathrm{p}}=0$ to make further understanding of the patterns presented in Fig.4. Each pitch in Fig.5(a) is corresponding to the cases of Fig.4(a). Fig.5(b) stand for the numerical calculations are on the focal plane. It is noticed that a dark region will be formed while combining the radial polarized beam with phase plate 1,3 and 4 . The hollow structure emerges in the modulation of azimuthal polarized beams with phase plate 1 and 4 as well.

Fig.6 Cross section of the axial symmetric high-intensity lobes of sub-diffraction-limit patterns. (a) topography of beam patterns on calculation plane; (b) position of calculation plane, $z_{p}$ is fluctuated around $0.9 \lambda$.
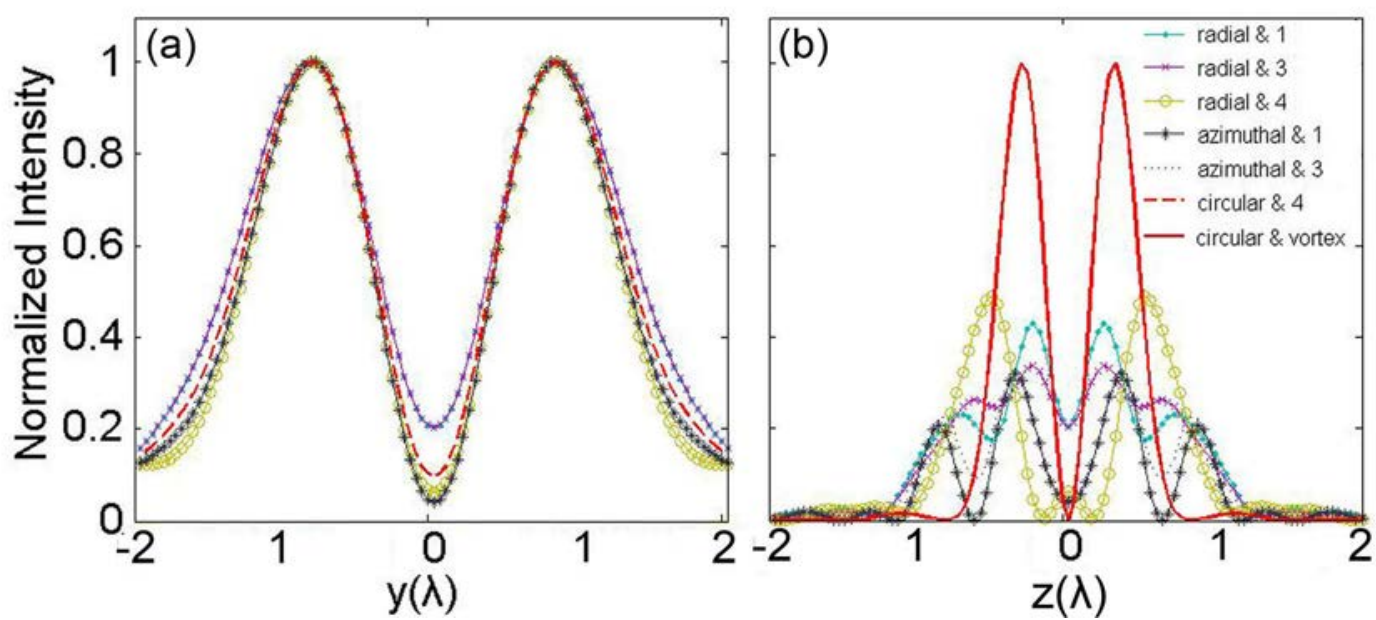

Fig. 7 Normalized intensity distributions on (a) xz plane $\left(\mathrm{z}_{\mathrm{py}}=0\right)$; $(\mathrm{b})$ xy plane $\left(\mathrm{z}_{\mathrm{p}}=0\right)$.

It is noticed that , the sub-diffraction-limit patterns have symmetric high-intensity lobes in axial direction. Fig.6 reveals the cross sections of the lobes corresponding to the cases in Fig.4. Since the peak intensity of the lobes are located differently in axial direction, the value of $z_{p}$ is different in the calculation. The calculation results, which are carried out by radial polarization with phase plate $1,3,4$, and azimuthal modulated by phase plate 1,3 , have shown that these structures are suit for improving the axial resolution in STED nanoscope. Particularly, a quite tightly focused patterns has been obtained by modulating the azimuthal polarized beam with Type 3 phase plate. The FWHM of the pattern is 0.424 folds of the incident wavelength. It is smaller than that of the diffraction limit pattern generated by focusing circular polarization beam without phase plate, of which the size is $0.432 \lambda$. These structures 
are also potential to be used to improve the performance in the optical tweezer as well.

The other beam patterns are also meaningful, such as the pattern obtained by type 2 . Two kinds of square shaped patterns with cross-shaped dark regions in their centers are created on the focal plane, while the patterns on the cross sections of the axial high-intensity lobes are hollow square and hollow cross respectively. There are two types of hollow-cylinder shaped nano patterns with the modulation of type 3 and 4 . These spatial sub-diffraction-limit patterns might be applied for the field of microfabrication, such as laser lithographing of special nano channels or pillars.

Fig.7 illustrates the normalized intensity of sub diffraction limitation patterns, which are acquired by radial polarization with phase plate $1,3,4$ and azimuthal polarized beam combined with phase plate 1,3 , on the $x z$ plane and xy plane. The intensity distributions of the beam patterns obtained by using vortex $2 \mathrm{Pi}$ and circular $\mathrm{Pi}$ are shown in Fig.7 as well. The maximum intensity of each beam patterns is normalized to 1. In Fig.7(a), it is obvious that the FWHM of these sub diffraction limit patterns in axial direction is very close to that of the circular polarized beam modulated by circular Pi plate. In Fig.7(b) the FWHM in the case of radial polarized beam through the phase plate 4 is bigger than that in the case of circular polarization modulated with vortex $2 \mathrm{Pi}$. While the others have a similar dimension compare to the case utilizing circular polarized beam and vortex $2 \mathrm{Pi}$ phase plate.

\section{Conclusion}

Based on the Vectorial Diffraction theory, some valuable sub-diffraction-limit patterns have been obtained by modulating the phase of different polarized beam with designed phase plates. The beams applied in the numerical simulation are circular, radial and azimuthal polarized Gaussian beams. The new kinds of phase plates are designed by combining the Circular Pi phase plate with Semicircle Pi, Quartered Pi and Vortex 2Pi phase plates. The mechanism of novel phase plates designing and special sub-diffraction-limit patterns creating is detailed revealed. By modulating radial and azimuthal polarized beam with two kinds of novel phase plates respectively, four kinds of sub-diffraction-limit patterns are acquired. The patterns are capable to compress the lateral and axial fluorescence PSF simultaneously in the far-filed STED nanoscope; these spatial sub-diffraction-limit hollow structures are also useful for stable capturing of the atom. This work shows a systematic numerical study of creating sub-diffraction-limit beam patterns with special wavefront designing of Guassian laser beam, which is significant for creating highperformance beam patterns for far-field nanoscope, optical tweezer, laser fabrication and etc.

\section{Acknowledgements}

This project is supported by the Young Scientist Fund of the National Natural Science Foundation of China (Grant No. 11504294), the Youth Talent Plan of the Natural Science Foundation of Shaanxi Province of China (Grant No. 2016JQ1030), the National Natural Science Foundation of China (Grant No. 61378083), the Natural Science Foundation of Shaanxi Province of China (Grant No. 2010JS110, 2013SZS03-Z01), the Major Research Plan of the National Natural Science Foundation of China (Grant No. 91123030), the International Cooperation Foundation of the National Science and Technology Major Project of the Ministry of Science and Technology of China (Grant No. 2011DFA12220).

\section{Reference}

[1] S. Chattopadhyay, Y. F. Huang, Y. J. Jen, A. Ganguly, K. H. Chen and L. C. Chen: Mater. Sci. Eng. R, 69, (2010) 1.

[2] H. C. Lo, H. I. Hsiung, S. Chattopadhyay, H. C. Han, C. F. Chen, J. P. Leu, K. H. Chen and L. C. Chen: Biosen. Bioelectron, 26, (2011) 2413.

[3] H. F. Yang, H. D. Hel, E. L. Zhao, J. B. Hao, J. G. Qian, W. Tang and H. Zhu: Laser Phys. Lett., 11, (2014) 105901.

[4] K. Takahiro, T. Yoshio, S. Noritsugu and H. Takuya: Phys. Rev. Lett., 78, (1997) 4713.

[5] S. W. Hell and J. Wichmann: Opt. Lett., 19, (1994) 780.

[6] T. A. Klar, S. W. Hell: Opt. Lett., 24, (1999) 954.

[7] V. Westphal, L. Kastrup, S. W. Hell,: Appl. Phys. B, 77, (2003) 377.

[8] V. Westphal and S. W. Hell: Physical Review Letters 94, (2005) 143903.

[9] E. Rittweger, K. Y. Han, S.E. Irvine, C. Eggeling and S. W. Hell: Nature Photonics, 3, (2009) 144.

[10] D. Wildanger, B. R. Patton, H. Schill, L. Marseglia, J. P. Hadden, S. Knauer, A. Schönle, J. G. Rarity, J. L. O'Brien, S. W. Hell and J. M. Smith J M: Adv. Materials, 24, (2012) 309.

[11]K. Sakai and S. Noda: Electronics Letters, 43, (2007) 107.

[12]C. Zhang, K. G. Wang, J. T. Bai, S. Wang, W. Zhao, F. Yang, C. Z. Gu and G. R. Wang: Nanoscale Research Letters, 8, (2013) 280.

[13] S. H. Deng, L. Li, Y. Cheng, R. X. Li and Z. Z. Xu: Optics Express, 18, (2010)1657.

[14]Q. Wang, X. W. Sun and P. Shum: Appl Opt. 43, (2004) 2292.

[15] S. Li, C. F. Kuang, X. Hao,Z. T. Gu and X. Liu: Journal of optics 14, (2012) 85704.

[16] W. Takeshi and I. Yasunori: Opt. Eng., 43, (2004) 1136.

[17] A. D. Jeffrey, L. L. Haavig and M. Don: Applied Optics, 36, (1997) 2376.

[18]W. Takeshi, M. Fujii, W. Yoshi and I. Yoshinori: Review of Scientific Instruments 75, (2004), 5131.

[19]X. M. Chen, C. J. Jin, L. F. Li, B. L. Lu,Z.Y. Ren and J. T. Bai: Optics Communication 316, (2014) 140.

[20]V. Sunil, K. Yuichi and S. Shunichi: Optics Express 21, (2013) 8972.

[21]R. K. Singh, P. Senthilkumaran and K. Singh: J. Opt. Soc. Am.: A 25, (2008)1307.

[22]R. K. Singh, P. Senthilkumaran and K. Singh: J. Opt. Soc. Am.: A 26, (2009) 576.

[23]Y. Miyamoto, K. Bito, Y. Ozaki, A. Wada, and M. Takeda: Opt. Rev. 20, (2013) 127.

[24]C. Zhang, K. G. Wang, S. Wang, Y. Liu, W. Zhao, X. M. Chen, B. L. Lu, Y. Sun, C. Z. Gu, G. R. Wang and J. T. Bai: J. Opt. 16, (2014) 125701.

[25]X. Hao, C. F. Kuang, T. T. Wang and X. Liu: Journal of Optics 12, (2010) 115707. 
[26]M. Born and E. Wolf:"Principles of Optics" ed. by M. Farley- Born and E. Wolf, (Publisher, Cambridge, 1999) p. 487.

(Received: May 26, 2016, Accepted: August 1, 2016) 